\title{
Predictors of in-hospital mortality following non- cardiac surgery: Findings from an analysis of a South African hospital administrative database
}

\author{
Y Moodley, MMedSci; B M Biccard, MB ChB, FCA (SA), FFARCSI, MMedSci, PhD \\ Perioperative Research Group, Department of Anaesthetics, College of Health Sciences, Nelson R Mandela School of Medicine, \\ University of KwaZulu-Natal and Inkosi Albert Luthuli Central Hospital, Durban, South Africa
}

Corresponding author: Y Moodley (moodleyyo@ukzn.ac.za)

\begin{abstract}
Background. Predictors of in-hospital mortality (IHM) following non-cardiac surgery in South African (SA) patients are not well described. Objective. To determine the association between patient comorbidity and IHM in a cohort of SA non-cardiac surgery patients.

Methods. Data related to comorbidity and IHM for 3727 patients aged $\geq 45$ years were obtained from a large administrative database at a tertiary SA hospital. Logistic regression analysis was used to determine independent predictors of IHM. In addition, population-attributable fractions (PAFs) were calculated for all clinical factors identified as independent predictors of IHM.

Results. Renal dysfunction, congestive heart failure, cerebrovascular disease, male gender and high-risk surgical specialties were independently associated with IHM (odds ratios (95\% confidence intervals) 7.585 (5.480 - 10.50); 2.604 (1.119 - 6.060); 2.645 (1.414 - 4.950 ); 1.433 (1.107 1.853); and $1.646(1.213-2.233)$, respectively). Ischaemic heart disease, diabetes and hypertension were not identified as independent predictors of IHM in SA non-cardiac surgery patients. Renal dysfunction had the largest contribution to IHM in this study (PAF 0.34), followed by high-risk surgical specialties (PAF 0.15), male gender (PAF 0.08), cerebrovascular disease (PAF 0.03) and congestive heart failure (PAF 0.03). Conclusion. Renal dysfunction, congestive heart failure, cerebrovascular disease, male gender and high-risk surgical specialties were major contributors to increased IHM in SA non-cardiac surgery patients. Prospectively designed research is required to determine whether ischaemic heart disease, diabetes and hypertension contribute to IHM in these patients.
\end{abstract}

S Afr Med J 2015;105(2):126-129. DOI:10.7196/SAMJ.8268

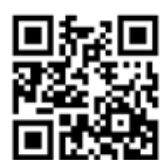

Weiser et al. ${ }^{[1]}$ estimated the global number of patients undergoing surgery in 2004 to be over 234 million, with $0.5 \%$ of these patients dying during or shortly after their surgical procedure. These alarming statistics emphasise the importance of surgical safety and the need to identify risk factors associated with in-hospital mortality (IHM) in patients before their surgery, so that these risk factors may be appropriately managed to reduce the risk of perioperative IHM. Studies of perioperative mortality have been confined primarily to European ${ }^{[2]}$ and North American ${ }^{[3]}$ surgical populations. Interestingly, the clinical importance of comorbidities in the pathology of adverse outcomes may differ between South African (SA) patients and patients from Europe and North America. ${ }^{[4]}$ The current epidemiological transition towards a higher burden of non-communicable disease in SA patients appears to explain these observed differences. ${ }^{[4,5]}$ For example, while hypertension has been associated with an almost four-fold increased risk of postoperative mortality in SA non-cardiac vascular surgery patients, ${ }^{[5]}$ similar research by a vascular surgery research group in New England, USA, did not identify hypertension as a predictor of adverse perioperative events. ${ }^{[6]}$ It is therefore imperative that SA non-cardiac patients be stratified for perioperative risk through a country-specific list of appropriate comorbid risk factors. We sought to determine the relationship between several common comorbid conditions and IHM in a cohort of SA non-cardiac surgery patients.

\section{Methods}

This study was conducted at Inkosi Albert Luthuli Central Hospital (IALCH), Durban, SA. The hospital provides a tertiary service to patients living in the province of KwaZulu-Natal.
Ethical approval was obtained from the Biomedical Research Ethics Committee of the University of KwaZulu-Natal before commencing the study. The hospital's administrative database was used to identify all patients aged $\geq 45$ years who underwent elective non-cardiac surgery at the hospital between 2005 and 2012. International Statistical Classification of Diseases and Related Health Problems (10th edition) (ICD-10) codes were then used to extract data related to comorbidities from patient electronic medical records and create a database for statistical analysis. We used an age of $\geq 45$ years as an inclusion criterion for this study, in keeping with other large studies of perioperative outcomes. ${ }^{[7]}$ The effects of the following comorbidities on IHM were investigated: hypertension, diabetes, renal dysfunction, ischaemic heart disease, congestive heart failure and cerebrovascular disease/stroke. Hypertension was included because it is highly prevalent in the SA surgical population. ${ }^{[5]}$ The other five comorbidities are established risk factors for poor perioperative outcomes and form part of Lee's Revised Cardiac Risk Index (RCRI), ${ }^{[8]}$ which is commonly used as a perioperative risk stratification tool at IALCH. In-hospital death was determined by evaluating each patient's discharge record following their surgery. Vascular surgery, thoracic surgery and general surgery were classified as high-risk surgical specialties. ${ }^{[2]}$ Duplicate records, patients aged $<45$ years, cardiac surgery patients and non-surgical patients were excluded from the final data set. Categorical data were analysed using the $\chi^{2}$ test or Fisher's exact test, where appropriate. Binary logistic regression was used to identify independent associations between comorbidities and IHM. A result was considered statistically significant at a $p$-value of $<0.05$. Results for the logistic regression analysis are presented as odds ratios (ORs) with $95 \%$ confidence intervals (CIs). The 
population-attributable fraction (PAF) for each factor independently associated with a higher incidence of IHM was also calculated. ${ }^{[9]}$ Univariate and multivariate statistical analyses were performed using the Statistical Package for the Social Sciences (SPSS) version 21 (SPSS Inc., USA).

\section{Results}

The final study cohort comprised 3727 non-cardiac surgery patients. Reasons for excluding patients from the final data set are shown in Fig. 1. Overall, the cumulative incidence of IHM in this study was $7.5 \%$. Table 1 shows the proportion of patients in the final study cohort by surgical specialty. Almost half of all patients underwent surgery that fell within a high-risk surgical specialty group. Renal surgery and vascular surgery were the surgical specialties associated with the highest levels of IHM (incidences of $24.3 \%$ and $7.0 \%$, respectively).

The preoperative clinical characteristics of the study cohort are shown in Table 2 . There was a high prevalence of diabetes and hypertension in the cohort $(46.7 \%$ and $79.1 \%$, respectively). Statistically significant univariate associations were observed between IHM and male gender $(p<0.001)$, elderly age $(p<0.001)$, diabetes $(p<0.001)$, cerebrovascular disease $(p=0.007)$ and renal dysfunction $(p<0.001)$ (Table 2).

Three comorbid conditions (renal dysfunction, congestive heart failure and cerebrovascular disease) were independently associated with an increased risk of IHM following non-cardiac surgery (Table 3). In addition, male gender and high-risk surgical specialties were also independently associated with an increased risk of IHM in this study (Table 3).
The results of the PAF analysis for each predictor of IHM are shown in Table 4. Renal

dysfunction had the largest contribution to IHM in this study (PAF 0.34), followed by

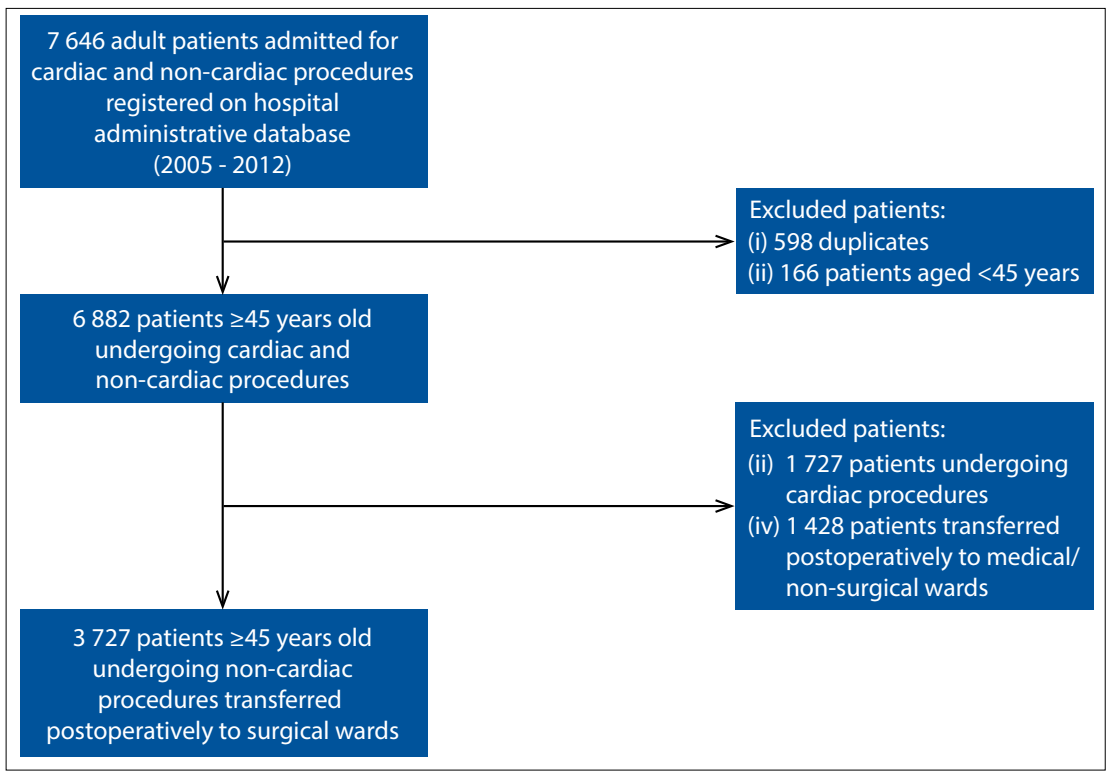

Fig. 1. Study profile.

\section{Table 1. Proportion of patients in the final study cohort by surgical specialty}

\begin{tabular}{ll}
\hline Surgical specialty & $\boldsymbol{n}$ (\% of final study cohort) \\
\hline Gynaecological surgery & $130(3.5)$ \\
General surgery & $481(12.9)$ \\
Vascular surgery & $1164(31.2)$ \\
Plastic surgery & $91(2.4)$ \\
Orthopaedic surgery & $321(8.6)$ \\
Urological surgery & $370(9.9)$ \\
Ear, nose and throat surgery & $96(2.6)$ \\
Renal surgery & $494(13.3)$ \\
Thoracic surgery & $162(4.4)$ \\
Ophthalmological surgery & $418(11.2)$ \\
Total & $3727(100.0)$
\end{tabular}

Table 2. Baseline patient clinical characteristics expressed as a frequency (\%)

\begin{tabular}{|c|c|c|c|c|}
\hline Patient characteristic & $\begin{array}{l}\text { Total cohort } \\
(N=3727) \\
n(\%)\end{array}$ & $\begin{array}{l}\text { Patients with } \\
\text { in-hospital mortality } \\
(N=278) \\
n(\%)\end{array}$ & $\begin{array}{l}\text { Patients without } \\
\text { in-hospital mortality } \\
(N=3449) \\
n(\%)\end{array}$ & $p$-value ${ }^{*}$ \\
\hline Male gender & $1591(42.7)$ & $150(54.0)$ & $1441(41.8)$ & $<0.001$ \\
\hline Age $>65$ years & $1265(33.9)$ & $65(23.4)$ & $1200(34.8)$ & $<0.001$ \\
\hline Ischaemic heart disease & $420(11.3)$ & $23(8.3)$ & $397(11.5)$ & 0.101 \\
\hline Diabetes & $1739(46.7)$ & $98(35.3)$ & $1641(47.6)$ & $<0.001$ \\
\hline Congestive heart failure & $50(1.3)$ & $7(2.5)$ & $43(1.2)$ & 0.095 \\
\hline Renal dysfunction & $487(13.1)$ & $119(42.8)$ & $368(10.7)$ & $<0.001$ \\
\hline Cerebrovascular disease/stroke & $87(2.3)$ & $13(4.7)$ & $74(2.1)$ & 0.007 \\
\hline Hypertension & $2948(79.1)$ & $209(75.2)$ & $2739(79.4)$ & 0.095 \\
\hline High-risk surgical specialty & $1807(48.5)$ & $125(45.0)$ & $1682(48.8)$ & 0.222 \\
\hline
\end{tabular}


Table 3. Results of multivariate analysis: clinical variables independently associated/ not independently associated with IHM

\begin{tabular}{lll}
\hline Clinical variable & OR $(\mathbf{9 5 \%}$ CI $)$ & $p$-value \\
\hline Male gender & $1.433(1.107-1.853)$ & 0.006 \\
Age $>65$ years & $0.840(0.616-1.147)$ & 0.273 \\
Ischaemic heart disease & $0.815(0.515-1.290)$ & 0.383 \\
Diabetes & $0.832(0.634-1.092)$ & 0.185 \\
Congestive heart failure & $2.604(1.119-6.060)$ & 0.026 \\
Renal dysfunction & $7.585(5.480-10.50)$ & $<0.001$ \\
Cerebrovascular disease/stroke & $2.645(1.414-4.950)$ & 0.002 \\
Hypertension & $1.119(0.823-1.522)$ & 0.474 \\
High-risk surgical specialty & $1.646(1.213-2.233)$ & $<0.001$ \\
${ }^{*}<<05$ was considered statistically significant. & &
\end{tabular}

Table 4. PAFs for clinical variables associated with IHM in SA non-cardiac surgery patients

\begin{tabular}{ll}
\hline Comorbidity & PAF \\
\hline Congestive heart failure & 0.03 \\
Renal dysfunction & 0.34 \\
Cerebrovascular disease/stroke & 0.03 \\
High-risk surgical specialty & 0.15 \\
Male gender & 0.08
\end{tabular}

high-risk surgery (PAF 0.15), male gender (PAF 0.08), cerebrovascular disease (PAF 0.03 ) and congestive heart failure (PAF 0.03).

\section{Discussion}

The incidence of postoperative IHM in our study was much higher than that reported for most Nothern and Western European countries ${ }^{[10]}$ This may be explained in part by the fact that almost half our patient population underwent surgery in a high-risk specialty, placing them at increased risk of IHM after the procedures.

We found univariate associations between increased IHM and male gender, age $>65$ years, diabetes, renal dysfunction, cerebrovascular disease and high-risk surgery. Interestingly, while our observation that renal dysfunction and cerebrovascular disease were more common in patients who died in hospital was in agreement with the studies of Pearse et al. ${ }^{[10]}$ Charlson et al. ${ }^{[11]}$ and Elixhauser et al. ${ }^{[12]}$ our observation that diabetes was significantly more common in patients who did not suffer IHM was in contrast to the findings of these studies.

Following multivariate statistical analysis, male gender, renal dysfunction, congestive heart failure, cerebrovascular disease and high-risk surgical specialties were identified as independent predictors of IHM in SA non-cardiac surgery patients. While the importance of renal dysfunction has been described in overseas surgical populations, its contribution to IHM in SA non-cardiac surgery patients is of concern, with just over one-third of the risk associated with IHM being attributed to renal dysfunction.

Waikar et al. ${ }^{[13]}$ found that although IHM rates among patients with acute renal dysfunction had decreased between 1988 and 2002, mortality rates for patients not requiring dialysis and those requiring dialysis were $>20 \%$ and $>30 \%$, respectively. In the perioperative setting, a serum creatinine level of $>177 \mu \mathrm{mol} / \mathrm{L}$ has previously been shown to be an independent predictor of perioperative cardiovascular morbidity and mortality in non-cardiac surgery patients. ${ }^{[8]}$ 'Renal disease' and 'renal failure' were also associated with IHM in the studies of Charlson et al ${ }^{[11]}$ and Elixhauser et al.. ${ }^{[12]}$ respectively.

In addition to the risk stratification studies of Charlson et al. ${ }^{[11]}$ and Elixhauser et al..$^{[12]}$ congestive heart failure was also identified as an independent predictor of cardiovascular morbidity and mortality in patients undergoing non-cardiac surgery by Lee et al., ${ }^{[8]}$ as well as in the validation study of Lee's RCRI. ${ }^{[14]}$ Hernandez et al. ${ }^{[15]}$ observed substantial levels of morbidity and mortality in older patients with congestive heart failure who underwent non-cardiac surgery. Similarly, Hammill et $a l .{ }^{[16]}$ found that patients with heart failure were at higher risk of operative mortality than those without (adjusted OR 1.63; 95\% CI 1.52 - 1.74).

It is estimated that up to $14 \%$ of patients with a first-time stroke may have stroke recurrence within a year. ${ }^{[17]}$ Patients with recurrent stroke have a higher mortality rate than those with a first-time stroke, ${ }^{[18]}$ with surgery and its associated physiological stresses likely to increase the risk of mortality. The findings of this study are once again in agreement with the studies by Lee et al..$^{[8]}$ and Charlson et al. ${ }^{[11]}$ who observed a higher risk of perioperative mortality in patients with a history of cerebrovascular disease.

High-risk surgical specialties were also found to be associated with an increased risk of IHM in our study, which is in agreement with a large database study of 3.7 million surgical procedures conducted in 102 Dutch hospitals by Noordzij et al. . $^{[2]}$

We did not find ischaemic heart disease, diabetes or hypertension to be independently associated with IHM in this study. It is likely that these comorbidities were under-diagnosed in our non-cardiac surgery population, in particular in the group of patients who suffered IHM. The problem of under-diagnosis of comorbidities has been established by a number of studies, ${ }^{[19-21]}$ although the reasons for underdiagnosis, as in our study, remain unclear. In addition, we did not observe elderly age to be an independent predictor of IHM following non-cardiac surgery in SA patients, although this finding may be related to the fact that only a third of our cohort was $>65$ years old.

\section{Study limitations}

This study was not without limitations. The identification of comorbidities in this study was based on a physician's diagnosis at admission and the subsequent coding of this diagnosis on the hospital administrative database. As such, we were unable to measure the extent of undiagnosed comorbidity in our study, which is likely to be an important determinant of the findings for ischaemic heart disease, diabetes and hypertension. Also, the administrative database was limited by the accuracy with which perioperative medication was recorded; we were therefore unable to investigate the impact of medication use on IHM following non-cardiac surgery. A prospectively designed study that includes appropriate measures to diagnose patient comorbidity, as well as perioperative medication use, is required.

\section{Conclusion}

The incidence of IHM following non-cardiac surgery in our study was much higher than that reported for several developed-world European countries. This finding highlights the importance of identifying independent predictors of IHM in non-cardiac surgery patients, so that attempts can be made to optimise a patient's condition prior to their surgery and reduce their risk of postoperative IHM.

Adequate preoperative management of renal dysfunction, congestive heart failure and cerebrovascular disease may improve outcome after surgery. It is likely that ischaemic heart disease, diabetes and hypertension are underdiagnosed in SA non-cardiac surgery patients. Further prospectively designed research is required to confirm our findings. 
Acknowledgements. This work forms a component of the doctoral studies of Y Moodley, who is the recipient of a doctoral scholarship awarded by the South African National Research Foundation.

\section{Referencew}

1. Weiser TG, Regenbogen SE, Thompson KD, et al. An estimation of the global volume of surgery: A modelling strategy based on available data. Lancet 2008;372(9633):139-144. [http://dx.doi. org/10.1016/S0140-6736(08)60878-8

2. Noordzij PG, Poldermans D, Schouten O, Bax JJ, Schreiner FA, Boersma E. Postoperative mortality in The Netherlands: A population-based analysis of surgery-specific risk in adults. Anesthesiology 2010;112(5):1105-1115. [http://dx.doi.org/10.1097/ALN.0b013e3181d5f95c]

3. Barnett S, Moonesinghe SR. Clinical risk scores to guide perioperative management. Postgrad Med J 2011;87(1030):535-541. [http://dx.doi.org/10.1136/pgmj.2010.107169]

4. Steyn K, Sliwa K, Hawken S, et al. Risk factors associated with myocardial infarction in Africa: The INTERHEART Africa study. Circulation 2005;112(23):3554-3561. [http://dx.doi.org/10.1161/
INT CIRCULATIONAHA.105.563452]

5. Biccard BM, Nepaul S. Risk factors associated with intermediate and long-term mortality following vascular surgery in South African patients. Cardiovasc J Afr 2010;21(5):263-267. [http://dx.doi.org/CVJ-21.004]

6. Bertges DJ, Goodney PP, Zhao Y, et al. The Vascular Study Group of New England Cardiac Risk Index (VSG-CRI) predicts cardiac complications more accurately than the Revised Cardiac Risk Index in vascular surgery patients. J Vasc Surg 2010;52(3):674-683. [http://dx.doi.org/10.1016/j.jvs.2010.03.031] 7. Devereaux PJ, Chan MT, Alonso-Coello P, et al. Association between postoperative troponin levels and 30-day mortality among patients undergoing noncardiac surgery. JAMA 2012;307(21):2295-2304. [http://dx.doi.org/10.1001/jama.2012.5502]

8. Lee TH, Marcantonio ER, Mangione CM, et al. Derivation and prospective validation of a simple index for prediction of cardiac risk of major noncardiac surgery. Circulation 1999;100(10):1043-1049. [http://dx.doi.org/10.1161/01.CIR.100.10.1043]

9. Steenland K, Armstrong B. An overview of methods for calculating the burden of disease due to specific risk factors. Epidemiology 2006;17(5):512-519. [http://dx.doi.org/10.1097/01. ede.0000229155.05644.43]
10. Pearse RM, Moreno RP, Bauer P, et al. Mortality after surgery in Europe: A 7 day cohort study. Lancet 2012;380(9847):1059-1065. [http://dx.doi.org/10.1016/S0140-6736(12)61148-9]

1. Charlson ME, Pompei P, Ales KL, MacKenzie CR. A new method of classifying prognostic comorbidity in longitudinal studies: Development and validation. J Chronic Dis 1987;40(5):373-383. [http://dx.doi. in longitudinal studies: Development
org/10.1016/0021-9681(87)90171-8]

12. Elixhauser A, Steiner C, Harris DR, Coffey RM. Comorbidity measures for use with administrative data. Med Care 1998;36(1):8-27

13. Waikar SS, Curhan GC, Wald R, McCarthy EP, Chertow GM Declining mortality in patients with acute ren failure, 1988 to 2002. J Am Soc Nephrol 2006;17(4):1143-1150. [http://dx.doi.org/10.1681/ASN.2005091017]

14. Boersma E, Kertai MD, Schouten O, et al. Perioperative cardiovascular mortality in noncardiac surgery: Validation of the Lee cardiac risk index. Am J Med 2005;118(10):1134-1141. [http://dx.doi. org/10.1016/j.amjmed.2005.01.064

15. Hernandez AF, Whellan DJ, Stroud S, Sun JL, O'Connor CM, Jollis JG. Outcomes in heart failure patients after major noncardiac surgery. J Am Coll Cardiol 2004;44(7):1446-1453. [http://dx.doi. org/10.1016/j.jacc.2004.06.059]

16. Hammill BG, Curtis LH, Bennett-Guerrero E, et al. Impact of heart failure on patients undergoing majo noncerdiacsurgery Anesthesiology 2008-108(4):559-567. [http.//dx doi org/10.1097/ALN 0b013e31816725ef] 7. Dickerson LM, Carek PI, Quattlebum RG. Prevention of recurrent ischemic stroke. Am Fam Physicin 2007:76(3):382-388.

18. Jorgensen HS, Nakayama H, Reith J, Raaschou HO, Olsen TS. Stroke recurrence: Predictors, severity and prognosis. The Copenhagen Stroke Study. Neurology 1997;48(4):891-895. [http://dx.doi org/10.1212/WNL.48.4.891]

19. Ashworth M, Lloyd D, Smith RS, Wagner A, Rowlands G. Social deprivation and statin prescribing A cross-sectional analysis using data from the new UK general practitioner 'Quality and Outcomes Framework. J Public Health (Oxf) 2007;29(1):40-47. [http://dx.doi.org/10.1093/pubmed/fdlo68]

20. Soljak M, Samarasundera E, Indulkar T, Walford H, Majeed A. Variations in cardiovascular disease under-diagnosis in England: National cross-sectional spatial analysis. BMC Cardiovasc Disord 2011;11:12. [http://dx.doi.org/10.1186/1471-2261-11-12]

21. Ward PR, Noyce PR, St Leger AS. Are GP practice prescribing rates for coronary heart disease drugs equitable? A cross sectional analysis in four primary care trusts in England. J Epidemiol Community Health 2004;58(2):89-96. [http://dx.doi.org/10.1136/jech.58.2.89]

Accepted 9 December 2014. 